\title{
Correction to: Circadian rhythm of COPD symptoms in clinically based phenotypes. Results from the STORICO Italian observational study
}

Nicola Scichilone ${ }^{1 *}$, Raffaele Antonelli Incalzi ${ }^{2}$, Francesco Blasi ${ }^{3}$, Pietro Schino ${ }^{4}$, Giuseppina Cuttitta ${ }^{5}$, Alessandro Zullo ${ }^{6}$, Alessandra Ori ${ }^{6}$, Giorgio Walter Canonica ${ }^{7}$ and on behalf of STORICO study group

\section{Correction to: Pulm Med (2019) 19:171 \\ https://doi.org/10.1186/s12890-019-0935-2}

Following publication of the original article [1], the authors flagged that the article had been provided with the names of the authors (not including the STORICO study group) in the wrong order: the 'Given Names' and Family Names' were erroneously swapped around.

Please see this table for the correct order of the names:

\begin{tabular}{ll}
\hline Family Name & Given name \\
\hline Scichilone & Nicola \\
Antonelli Incalzi & Raffaele \\
Blasi & Francesco \\
Schino & Pietro \\
Cuttitta & Giuseppina \\
Zullo & Alessandro \\
Ori & Alessandra \\
Canonica & Giorgio Walter \\
\hline
\end{tabular}

Policlinico and Department of Pathophysiology and Transplantation, University of Milan, via Francesco Sforza, 35, 20122 Milan, Italy. ${ }^{4}$ Miulli Hospital, Acquaviva delle FontiStrada Prov. 127 Acquaviva - Santeramo Km. 4, 10070021 Bari, Italy. ${ }^{5}$ National Research Council, via Ugo La Malfa, 153, 90146 Palermo, Italy. ${ }^{6}$ Medineos Observational Research, Viale Virgilio 54/U, 41123 Modena, Italy. ${ }^{7}$ Personalized Medicine Asthma and Allergy Clinic Humanitas University Humanitas research Hospital Rozzano (Milan), via Manzoni, 56, 20089 Rozzano, Ml, Italy.

Published online: 04 December 2019

\section{Reference}

1. Scichilone $\mathrm{N}$, et al. Circadian rhythm of COPD symptoms in clinically based phenotypes. Results from the STORICO Italian observational study. Pulm Med. 2019;19:171. https://doi.org/10.1186/s12890-019-0935-2.

This error has now been corrected in the original article and the corrected author list is detailed in this article.

\footnotetext{
Author details

${ }^{1}$ DIBIMIS, University of Palermo, Piazza delle Cliniche, 2, 90127 Palermo, Italy. ${ }^{2}$ University Biomedical Campus of Rome, via Alvaro del Portillo, 21, 00128 Rome, Italy. ${ }^{3}$ Internal Medicine Department, Respiratory Unit and Cystic Fibrosis Adult Center Fondazione IRCCS Cà Granda Ospedale Maggiore

The original article can be found online at https://doi.org/10.1186/s12890019-0935-2

*Correspondence: nicola.scichilone@unipa.it; medidata@medineos.com

'DIBIMIS, University of Palermo, Piazza delle Cliniche, 2, 90127 Palermo, Italy

Full list of author information is available at the end of the article
}

(c) The Author(s). 2019 Open Access This article is distributed under the terms of the Creative Commons Attribution 4.0 International License (http://creativecommons.org/licenses/by/4.0/), which permits unrestricted use, distribution, and reproduction in any medium, provided you give appropriate credit to the original author(s) and the source, provide a link to the Creative Commons license, and indicate if changes were made. The Creative Commons Public Domain Dedication waiver (http://creativecommons.org/publicdomain/zero/1.0/) applies to the data made available in this article, unless otherwise stated. 